\title{
Asymptotic solutions of the Helmholtz equation: generalised Friedlander-Keller ray expansions of fractional order
}

\author{
R H Tew \\ School of Mathematical Sciences \\ University Park \\ University of Nottingham \\ Nottingham NG7 2RD \\ UK
}

May 24, 2018

Key Words: Ray theory; WKBJ-method

\begin{abstract}
Applications of a WKBJ-type 'ray ansatz' to obtain asymptotic solutions of the Helmholtz equation in the high-frequency limit are now standard, and underpin the construction of 'geometrical optics' ray diagrams in many electromagnetic, acoustic and elastic reflection, transmission and other scattering problems. These applications were subsequently extended by Keller to include other types of rays - called 'diffracted' rays - to provide an accessible and impressively accurate theory which is relevant in wide-ranging sets of circumstances. Friedlander and Keller then introduced a modified ray ansatz to extend yet further the scope of ray theory and its applicability to certain other classes of diffraction problems (tangential ray incidence upon an obstructing boundary, for instance), and did so by the inclusion of an extra term proportional to a power of the wavenumber within the exponent of the initial ansatz.

Our purpose here is to generalise this further still by the inclusion of several such terms, ordered in a natural sequence in terms of strategically-chosen fractional powers of the large wavenumber, and to derive a systematic sequence of boundary value problems for the coefficient phase functions that arise within this generalised exponent, as well as one for the leading-order amplitude occurring as a pre-exponential factor.

One particular choice of fractional power is considered in detail, and waves with specified radially-symmetric or planar wavefronts are then analysed, along with a boundary value problem typifying two-dimensional radiation whereby arbitrary phase and amplitude variations are specified on a prescribed boundary curve. This theory is then applied to the scattering of plane and cylindrical waves at curved boundaries with small-scale perturbations to their underlying profile.
\end{abstract}




\section{Introduction and motivation}

There are two principal aims that motivate the work presented here, the first being to further develop the method introduced by Friedlander and Keller [1], who extended the breadth of ray-type solutions to the Helmholtz equation

$$
\left(\nabla^{2}+k^{2}\right) \phi=0
$$

in the singularly-perturbed, high-frequency limit $k \rightarrow \infty$. The second is to then apply this current development to determine the phase and amplitude structure of the scattered fields arising when incoming monochromatic waves are incident upon curved boundaries of arbitrary profile but with small-scale perturbations superimposed upon them. Within this general setting, examples of practical applications are plentiful, and $\phi$ might represent a potential function associated with an acoustic or elastic wave disturbance or else perhaps a component of a propagating electromagnetic field, amongst other possibilities.

To present a theoretical motivation for this current study, we return to basics and note that it is normal to decompose the wave function $\phi$ into an amplitude $A(\boldsymbol{x} ; k)$ and a phase $S(\boldsymbol{x} ; k)$ by writing

$$
\phi=A e^{i S}
$$

throughout the calculations that follow both $A$ and $S$ are allowed to be complex-valued, with the linearity of (1) permitting real or imaginary parts of $\phi$ to be taken as the final step in order to obtain a physically relevant solution. Notice that, at this stage, both $A$ and $S$ carry the large wavenumber $k$ as a parameter and much of the detail in what follows depends upon the precise nature of this dependence.

Formal substitution of (2) into (1) yields a modified (but as yet still exact) field equation coupling $A$ and $S$ in the form

$$
\nabla^{2} A+2 i \nabla A \cdot \nabla S+\left(k^{2}-|\nabla S|^{2}+i \nabla^{2} S\right) A=0
$$

in which $|\nabla S|^{2}=\nabla S \cdot \nabla S$. Given that the limit $k \rightarrow \infty$ in (1) is singularly-perturbed, a key objective in the WKBJ-type analysis to follow is to balance the ' $k^{2}$ '-term in (3) with at least some of the other terms present and a natural choice - one that underpins the classical ray theory of mathematical optics [2], [3] and the geometrical theory of diffraction (GTD) $[4]$ - is to set

$$
S(\boldsymbol{x} ; k)=k u(\boldsymbol{x}),
$$

where the 'phase function' $u$ is independent of $k$ and where we assume that the amplitude $A$ is at most algebraic in $k$ in a manner such that

$$
A(\boldsymbol{x} ; k) \sim A_{0}(\boldsymbol{x})+O\left(k^{-1}\right) \quad \text { as } \quad k \rightarrow \infty,
$$

with $\left|A_{0}\right|=O(1)$. Under these conditions, the largest terms in (3) are necessarily those of $O\left(k^{2}\right)$, and picking these off leads directly to the eikonal equation

$$
|\nabla u|^{2}=1
$$


for $u$ alone, and since this equation has de-coupled entirely from $A$ we can go ahead and determine $u$ in isolation from the rest of the calculation, feeding its solution into subsequent analyses wherever it is needed. Notice also that in this particular case, the term $\left(k^{2}-(\nabla S)^{2}\right) A$ in (3) now disappears completely, leaving behind a modified (and still, as yet, exact) field equation

$$
\nabla^{2} A+2 i k \nabla A \cdot \nabla u+i k A \nabla^{2} u=0
$$

This feature is true only for the specific choice for $S$ in (4) and is not true for subsequent choices to follow.

Since $u$ can now be considered as already determined, (7) is to be treated as a partial differential equation for $A$, and the stipulations contained in (5) and the linearity in $k$ of (7) guides us to write

$$
A(\boldsymbol{x} ; k)=\sum_{n=0}^{\infty} \frac{A_{n}(\boldsymbol{x})}{(i k)^{n}},
$$

in which each $A_{n}(n=0,1,2, \ldots)$ is independent of $k$ and the factor $i^{-n}$ is included solely for algebraic convenience. Substitution of (8) into (7) and isolation of all terms of like order in $k^{-n}$ now yields the recursive system of 'transport equations' for the leading-order $\left(A_{0}\right)$ and successive $\left(A_{n}, n=1,2,3 \ldots\right)$ amplitudes

$$
\begin{aligned}
2 \nabla A_{0} \cdot \nabla u+A_{0} \nabla^{2} u & =0 \\
2 \nabla A_{n+1} \cdot \nabla u+A_{n+1} \nabla^{2} u+\nabla^{2} A_{n} & =0, \quad n=0,1,2, \ldots .
\end{aligned}
$$

It turns out that the solution for $u$ is, given appropriate boundary data together with any 'radiation' or 'outgoing-wave' conditions at infinity, amenable to an exact characteristic analysis via Charpit's method yielding the 'rays' of geometrical diffraction theories, and then the transport equations (9) and (10) reduce to first-order ordinary differential equations along them which, in turn, are straightforward to solve [5], [6].

This procedure provides the mathematical background to Keller's geometrical theory of diffraction, in which recipes (all geometrically based, and including associated phase and amplitude variations) for new families of rays engendered when an incoming ray impinges an obstruction at a point of reflection, transmission or diffraction are laid out (see Keller et al [6], [7] for details of specific examples, whilst applications relevant to electromagnetism and elasticity in particular are provided by Molinet et al [8] and Achenbach et al [9], respectively).

However, it soon became clear that the decomposition (4) was not sufficient to accommodate all wave phenomena that this theory predicted. One example occurs when an incoming ray is incident along a tangent to an obstructing, convex boundary, initiating the excitation of a radiative 'creeping' wave which then propagates around the continuation of the boundary from the point of ray tangency ([10], [11], [12], [13], [14]). Other examples include amplitude focussing associated with the formation of a caustic within a reflected 
or diffracted field ([15], [16]) and the analyses of various 'transition regions' arising in the scattering of electromagnetic waves at curved, plane gratings [17]. In these cases, variable terms proportional to $k^{1 / 3}$ arise in the phase structure in addition to the original term linear in $k$, so that broadly speaking (4) has to be modified to

$$
S(\boldsymbol{x} ; k)=k u(\boldsymbol{x})+k^{1 / 3} v(\boldsymbol{x}),
$$

together with a modification to the amplitude expansion (8) associated with it (the expansion is now in terms of inverse powers of $k^{1 / 3}$, rather than $k$, so that a general term in the modified expansion is proportional to $k^{-n / 3}$ ) .

To accommodate this possibility, Friedlander and Keller [1] considered a modified class of asymptotic solutions to the Helmholtz equation (1) in the form

$$
\phi \sim e^{i k u-k^{\alpha} v} \sum_{n=0}^{\infty} A_{n} k^{-\lambda_{n}} \quad \text { as } \quad k \rightarrow \infty,
$$

where we note that the notation that we have used in (12) above is different to that adopted by Friedlander and Keller (the roles of $\phi$ and $u$ are exactly interchanged, their $\chi$ becomes our $v$ and their $v_{n}$ 's (and, more generally, $v$ ), become our $A_{n}$ 's (and $A$, respectively); notice also that the $A_{n}$ 's in (12) are now also different to those used in (8)). Insofar as the phase is concerned, this is equivalent to setting

$$
S(\boldsymbol{x} ; k)=k u(\boldsymbol{x})+i k^{\alpha} v(\boldsymbol{x}),
$$

with $S$ being modified from (4) by the inclusion of the extra term proportional to $k^{\alpha}$.

One of Friedlander and Keller's main results was that the only instance when the transport equation for the leading-order amplitude $A_{0}$ deviated away from (9) was when $\alpha=1 / 2$, in which case it is (using our notation in (12)) modified to

$$
2 \nabla A_{0} \cdot \nabla u+\left(\nabla^{2} u+i \nabla u \cdot \nabla v\right) A_{0}=0
$$

and this case was analysed in the current context in a prequel to this paper [18] where, as here, we modified Friedlander and Keller's original ansatz slightly by inserting a multiplicative factor $i$ in the $k^{\alpha}$ term within the exponent (a step which makes virtually no procedural difference but which makes the ansatz directly applicable to problems considered in that paper involving wave scattering from certain classes of boundary).

In light of the fact that this one value of $\alpha$ alone gave rise to a different transport equation for $A_{0}$ we termed it a 'canonical' case, but it turns out that this choice is not the main reason why this is so; it has more to do with the fact that there is just one extra term (other than the term proportional to $u(\boldsymbol{x})$ and linear in $k)$ within the exponent. Had there been any other additional terms there, perhaps proportional to some other power of $k$, then this change of leading-order transport equation would have been automatic for all choices of $\alpha$. 
Hence, there are natural extensions of the work of Friedlander and Keller not considered so far, and this is a major motivation for the current work. As we have already noted at the start of this introduction, another lies in the applications we have in mind, which are themselves extensions of the wave scattering problems involving perturbed boundaries considered in [18]. The solution methodology is summarised in the Discussion section at the end of this current paper, though we outline here the statement of the problem to show why the suggested modifications to Friedlander and Keller's original theory [1] are necessary, and this outline is as follows.

Suppose we consider the scattering of a two-dimensional, cylindrically-spreading wave emanating from a source located at the Cartesian point $(0, h)$ well removed from a perturbed boundary. Suppose also that this incoming wave has the usual $R^{-1 / 2}$ leading-order algebraic decay rate and a given angular 'directivity' profile $F_{0}(\Theta)$, where $(R, \Theta)$ are plane polar coordinates centred on the source (so that $R=\left[x^{2}+(y-h)^{2}\right]^{1 / 2}$ ). Given this information, the asymptotic expansion of this incident field as $k \rightarrow \infty$ is given by

$$
\phi^{i n c}(R, \Theta ; k) \sim e^{i k R} \sum_{n=0}^{\infty} F_{n}(\Theta)(k R)^{-(n+1 / 2)},
$$

where the directivity functions $F_{n}(\Theta)$ follow recursively from $F_{0}(\Theta)$ via the relations

$$
F_{n+1}(\Theta)=-\frac{i F_{n}(\Theta)}{2(n+1)}\left[(n+1 / 2)^{2}+\frac{F_{n}^{\prime \prime}(\Theta)}{F_{n}(\Theta)}\right] \text {. }
$$

Suppose now that the obstructing boundary has Cartesian profile $y=k^{-1 / N} f(x)$ for some positive integer $N$, and that this boundary is to be interpreted here as a small perturbation from the $x$-axis. Regardless of the nature of the boundary condition imposed upon the reflecting boundary (Neumann or Dirichlet, for example), any ansatz of the form (2) for the reflected field will inevitably inherit the phase structure of the incident field at the boundary itself, and under more usual circumstances this condition would have yielded Snell's law of reflection, for example. Hence, in this case we have that on the boundary the phase of the reflected field is given by

$$
\begin{aligned}
S & =k\left[x^{2}+\left(k^{-1 / N} f(x)-h\right)^{2}\right]^{1 / 2} \\
& \sim k\left(x^{2}+h^{2}\right)^{1 / 2} \\
& +\sum_{n=1}^{N} \sum_{p=0}^{N-n} \frac{2^{p+n-N}}{(N-p-n) !(p+2 n-N) !}\left(\prod_{j=1}^{n}(3-2 j)\right) \frac{(-f(x))^{N-p}}{\left(x^{2}+h^{2}\right)^{n-1 / 2}} h^{p+2 n-N} k^{p / N} \\
& +O\left(k^{-1 / N}\right)
\end{aligned}
$$

and it is immediately clear by comparing (17) with (13) that the modification of $S$ away from (4) by the inclusion of just one extra term is not sufficient. In fact, after some algebra, 
(17) can be re-expressed in the alternative form

$$
S \sim \sum_{m=1}^{N} S_{m}(x) k^{m / N}+O\left(k^{-1 / N}\right) \quad \text { on } \quad y=k^{-1 / N} f(x)
$$

with $S_{N}(x)=\left(x^{2}+h^{2}\right)^{1 / 2}$, and this strongly suggests a form for $S$ with $N$ terms altogether (neglecting all terms of order $k^{-1 / N}$ and smaller). Further, these $N$ terms comprise a sequence in $m$, each term being a variable proportional to $k^{m / N}$ for $m=1,2, \ldots N$. This, in turn, suggests that the first term (involving $u$ ) on the right-hand-side of (13) should be associated with the final $(m=N)$ term of our sequence, and the single term on that same right-hand-side be replaced by an entire sum of $N-1$ terms involving $k^{1 / N}, k^{2 / N}, \ldots$, $k^{(N-1) / N}=k^{1-1 / N}$, and this comment motivates our choice of definition for a generalised Friedlander-Keller ray expansion of fractional order. Before proceeding with this definition and ensuing details for general values of $N$, followed by specific examples for the case $N=3$, we remark that there is one and only one instance when this generalised notion and the original idea of Friedlander and Keller coincide, and that is precisely when $\alpha=1 / 2$. That is to say, had we chosen a different value, $\alpha=1 / 3$ say, then while the original ansatz would comprise just two terms in the exponent, one proportional to $k$ and the other to $k^{1 / 3}$, our idea here would be to consider all powers of $k$ between and including $k^{1 / 3}$ and $k$, not just two outer limits, which would amount to the insertion of an extra term (just one in this case), proportional to $k^{2 / 3}$. If $\alpha=1 / 2$, there are no extra terms to include even if you wanted to, and the two theories are exactly the same.

The example cited above exemplifies the need for the inclusion of all such intermediate fractional powers of $k$ within the exponent of the ansatz, but that does not necessarily mean that they are all actually present in the final solution of any one particular problem. For example, the cases of creeping waves and caustics already cited essentially has $\alpha=1 / 3$, and yet somewhat mysteriously there are no terms involving $k^{2 / 3}$ in the exponents of the solution to either problem. Had there been, the contents of the current paper would no doubt have been investigated at that time.

\section{Generalised Friedlander-Keller ray expansions of fractional order}

\subsection{Definitions for general fractional exponent}

In all that follows we concentrate on fractional values of $\alpha$, though the theory of Friedlander and Keller does not require this restriction. When talking about setting $\alpha=1 / N$ for some positive integer $N$, our motivating example in the previous section suggests we adopt the convention that the associated Friedlander-Keller ansatz should have all powers of the form 
$k^{m / N}, m=1,2, \ldots N$ within its exponent, prompting us to write

$$
S(\boldsymbol{x} ; k)=\sum_{m=1}^{N} v_{m}(\boldsymbol{x}) k^{m / N}
$$

and

$$
A(\boldsymbol{x} ; k)=\sum_{n=0}^{\infty} A_{n}(\boldsymbol{x}) k^{-n / N}
$$

with the understanding that the $A_{n}$ 's occurring in (20) are not the same as those in (8) (though we do remark that the standard theory described by equations (4)-(10) is encapsulated in this revised approach by setting $N=1, v_{1}(\boldsymbol{x})=u(\boldsymbol{x})$ and multiplying $A_{n}(\boldsymbol{x})$ in (20) by $i^{-n}$.)

We shall refer to this revised ansatz as a Friedlander-Keller ray ansatz of fractional order $1 / N$, to the functions $v_{m}(\boldsymbol{x}), m=1,2, \ldots, N$ as the phase functions and $A_{n}(\boldsymbol{x}), n=0,1,2, \ldots$ as the amplitude functions. We shall consider any particular problem to be solved to leading order once each $v_{m}$ and $A_{0}$ have been determined.

In the previous analysis based around the choice (4) for $S$, the term $k^{2}-|\nabla S|^{2}=$ $k^{2}\left[1-|\nabla u|^{2}\right]$ was completely removed from (3), with many simplifying consequences for the ensuing analysis, once the choice (6) is made. However, this is no longer true in the current situation because the summation of terms in (19) implies a sequence of cross-product terms in $|\nabla S|^{2}$, all of which must be accounted for separately. In fact, after manipulation we have that

$$
\begin{aligned}
k^{2}-|\nabla S|^{2} & =\sum_{p=1}^{N}\left(\delta_{p N}-\sum_{j=p}^{N} \nabla v_{j} \cdot \nabla v_{N+p-j}\right) k^{1+p / N} \\
& -\sum_{p=2}^{N}\left(\sum_{j=1}^{p-1} \nabla v_{j} \cdot \nabla v_{p-j}\right) k^{p / N}
\end{aligned}
$$

in which $\delta_{i j}$ is the Kronecker delta tensor, and where we have separated out the sums so that the first sequence of summations contains all powers of $k$ larger than one and the second all terms of $O(k)$ and smaller. The reason for splitting the terms like this is because we can see that when (19) and (20) are substituted into (3), all terms other than those from $k^{2}-(\nabla S)^{2}$ are at most $O(k)$ without anything to balance each of the $N$ terms in the first sum in (21). Since each of these terms carries a different power of $k\left(k^{1+p / N}, p=1,2, \ldots N\right.$, in fact) then we deduce that they must each vanish identically on an individual basis, leading to a sequence of coupled (bar one) partial differential equations for the phase functions in the recursive format

$$
\delta_{p N}-\sum_{j=p}^{N} \nabla v_{j} \cdot \nabla v_{N+p-j}=0, \quad p=N, N-1, N-2, \ldots, 1 .
$$

These $N$ equations, all decoupled from each $A_{n}$, are to be solved to determine the $N$ phase functions $v_{N}, v_{N-1}, v_{N-2}, \ldots, v_{1}$. Also, it is now the case that the first sum in (21) vanishes 
altogether, leaving just the second one to contribute to (3). In fact, the resulting equation in full is

$$
\begin{aligned}
\sum_{n=0}^{\infty}\left(\nabla^{2} A_{n}\right) k^{-n / N} & +2 i \sum_{m=1}^{N} \sum_{n=0}^{\infty} \nabla v_{m} \cdot \nabla A_{n} k^{(m-n) / N}-\sum_{p=2}^{N} \sum_{j=1}^{p-1} \sum_{n=0}^{\infty}\left(\nabla v_{j} \cdot \nabla v_{p-j}\right) A_{n} k^{(p-n) / N} \\
& +i \sum_{m=1}^{N} \sum_{n=0}^{\infty}\left(\nabla^{2} v_{m}\right) A_{n} k^{(m-n) / N}=0
\end{aligned}
$$

Notice that the biggest term in the first (single) summation is $O(1)$, and will not contribute at all until the $N$ terms of $O\left(k^{1 / N}\right), O\left(k^{2 / N}\right), \ldots, O\left(k^{N / N}\right)=O(k)$ present in the other terms have been dealt with. We do not list all of the various possibilities but instead record ( $i$ ) the results once all terms contribute and $(i i)$ the transport equation for the leading-order amplitude $A_{0}$, these being

$$
\begin{aligned}
\nabla^{2} A_{r} & +i\left(2 \nabla v_{1} \cdot \nabla A_{r+1}+\left(\nabla^{2} v_{1}\right) A_{r+1}\right) \\
& +\sum_{p=2}^{N}\left[i\left(2 \nabla v_{p} \cdot \nabla A_{p+r}+\left(\nabla^{2} v_{p}\right) A_{p+r}\right)-\left(\sum_{j=1}^{p-1} \nabla v_{j} \cdot \nabla v_{p-j}\right) A_{p+r}\right]=0,
\end{aligned}
$$

obtained from the $O\left(k^{-r / N}\right)$ terms of $(23)$, for $(i)$ and

$$
2 i \nabla v_{N} \cdot \nabla A_{0}+A_{0}\left[-\sum_{j=1}^{N-1}\left(\nabla v_{j} \cdot \nabla v_{N-j}\right)+i \nabla^{2} v_{N}\right]=0
$$

for $(i i)$, respectively. Notice that (24) is one of a recursive system of partial differential equations (this one for $A_{N+r}$ ) kick-started by the solution for $A_{0}$ obtained from (25). In fact, we can consider (24) as generic provided we interpret $A_{-j}(\boldsymbol{x}) \equiv 0, j=N, N-1, \ldots, 1$ and then (25) follows by setting $r=-N$.

\subsection{The eikonal equation}

If we set $p=N$ into (22) then we obtain the eikonal equation

$$
\nabla v_{N} \cdot \nabla v_{N}=1
$$

for $v_{N}$, an equation which has entirely decoupled from any dependence on any of the other field variables in the problem and a result which is true for general $N$. Because of this generality, it is worth expanding a little beyond the reference to Charpit's method of solution already made by noting that if, as in that method, we introduce characteristic curves $\Gamma$ defined in terms of a parameter $\tau$ and the as yet unknown function $v_{N}$ by

$$
\frac{d \boldsymbol{x}}{d \tau}=\nabla v_{N}
$$

then: 
1. The eikonal equation (26) is equivalent to

$$
\frac{d \boldsymbol{x}}{d \tau} \cdot \frac{d \boldsymbol{x}}{d \tau}=1,
$$

so that $\tau$ is arc-length measured along $\Gamma$.

2. Since $\nabla\left(\nabla v_{N} \cdot \nabla v_{N}\right)=\mathbf{0}$, we immediately also have that $\left(\nabla v_{N} \cdot \nabla\right)\left(\nabla v_{N}\right)=\mathbf{0}$ and hence that $\left(\frac{d \boldsymbol{x}}{d \tau} \cdot \nabla\right)\left(\nabla v_{N}\right)=\mathbf{0}$ along $\Gamma$. This is, in turn, equivalent to

$$
\frac{d}{d \tau}\left(\nabla v_{N}\right)=\mathbf{0} \quad \text { along } \quad \Gamma .
$$

Given the defining equation (27), this implies that each characteristic, or 'ray', $\Gamma$ is a straight line.

3. The eikonal equation (26) is also equivalent to

$$
\frac{d \boldsymbol{x}}{d \tau} \cdot \nabla v_{N}=1
$$

and this becomes the ordinary differential equation $\frac{d v_{N}}{d \tau}=1$ along $\Gamma$. Hence we can now solve for $v_{N}$ along the rays, the solution being $v_{N}=v_{N}^{(0)}+\tau$ where $v_{N}^{(0)}$ is some given data at the location $\tau=0$ on $\Gamma$, and this location is a curve (in two dimensions) or surface (in three) on which data for the eikonal function $v_{N}$ is prescribed.

4. An important geometrical aspect of this concerns the wavefronts, defined by the condition $v_{N}(\boldsymbol{x}) \equiv$ constant. Since the normal to the surface defined by this condition is necessarily in the direction of $\nabla v_{N},(27)$ then shows that the rays are everywhere locally normal to the wavefronts, so knowing the geometry of either the rays or the wavefronts automatically implies that of the other.

Returning to the third point in the list above, suppose that data for $v_{N}$ is given on the surface $S$ defined in terms of two independent surface coordinates $s_{1}$ and $s_{2}$ by $S: \boldsymbol{x}=\boldsymbol{x}_{\mathbf{0}}\left(s_{1}, s_{2}\right)$, so that $v_{N}=v_{N}^{(0)}\left(s_{1}, s_{2}\right)$ and $\tau=0$ on $S$. Since we know that $\nabla v_{N}$ is conserved along the rays, we can now integrate (27) along them, subject to their emanating from this boundary $S$ at $\tau=0$, to obtain their straight line equation in the vectorial form

$$
\boldsymbol{x}=\boldsymbol{x}_{\mathbf{0}}\left(s_{1}, s_{2}\right)+\tau \nabla v_{N},
$$

along which the formula quoted above now yields $v_{N}(s, \tau)=v_{N}^{(0)}\left(s_{1}, s_{2}\right)+\tau$. All we now need do to finalise the solution is to evaluate $\nabla v_{N}$, and even then all we need is its evaluation on the boundary $S$ (since that value is then propagated along the rays). 
To do this, we note that $\frac{\partial \boldsymbol{x}_{\mathbf{0}}}{\partial s_{1}}, \frac{\partial \boldsymbol{x}_{\mathbf{0}}}{\partial s_{2}}$ and $\frac{\partial \boldsymbol{x}_{\mathbf{0}}}{\partial s_{1}} \times \frac{\partial \boldsymbol{x}_{\mathbf{0}}}{\partial s_{2}}$ are three linearly-independent vectors, allowing us to write

$$
\nabla v_{N}=\alpha_{1} \frac{\partial \boldsymbol{x}_{\mathbf{0}}}{\partial s_{1}}+\alpha_{2} \frac{\partial \boldsymbol{x}_{\mathbf{0}}}{\partial s_{2}}+\alpha_{3} \frac{\partial \boldsymbol{x}_{\mathbf{0}}}{\partial s_{1}} \times \frac{\partial \boldsymbol{x}_{\mathbf{0}}}{\partial s_{2}}
$$

where each $\alpha_{i}=\alpha_{i}\left(s_{1}, s_{2}\right), i=1,2,3$. Of these coefficient functions, $\alpha_{1}$ and $\alpha_{2}$ are then easily calculated explicitly by considering the scalar product of (32) with each of $\frac{\partial \boldsymbol{x}_{\mathbf{0}}}{\partial s_{1}}$ and $\frac{\partial \boldsymbol{x}_{\mathbf{0}}}{\partial s_{2}}$ in turn and forming a system of two linear, simultaneous equations for $\alpha_{1}$ and $\alpha_{2}$, the left-hand-sides (given the way we have laid the above expressions out) to which are known by differentiating the given boundary data $v_{N}=v_{N}^{(0)}\left(s_{1}, s_{2}\right)$ on $S$ as follows:

$$
\frac{\partial v_{N}^{(0)}}{\partial s_{i}}=\nabla v_{N} \cdot \frac{\partial \boldsymbol{x}_{\mathbf{0}}}{\partial s_{i}}, \quad i=1,2,
$$

this procedure being greatly simplified if $s_{1}$ and $s_{2}$ are chosen to be orthogonal coordinates, so that $\frac{\partial \boldsymbol{x}_{\mathbf{0}}}{\partial s_{1}} \cdot \frac{\partial \boldsymbol{x}_{\mathbf{0}}}{\partial s_{2}}=0$ and even simpler still if $s_{1}$ and $s_{2}$ are additionally chosen to be arc-length along their coordinate directions (so that $\frac{\partial \boldsymbol{x}_{\mathbf{0}}}{\partial s_{i}} \cdot \frac{\partial \boldsymbol{x}_{\mathbf{0}}}{\partial s_{j}}=\delta_{i j}, i, j=1,2$, and hence $\left.\alpha_{i}=\nabla v_{N} \cdot \frac{\partial \boldsymbol{x}_{\mathbf{0}}}{\partial s_{i}}=\frac{\partial v_{N}^{(0)}}{\partial s_{i}}, i=1,2\right)$.

The third coefficient $\alpha_{3}$ in (32), and hence $\nabla v_{N}$, is then determined (given the solutions for $\alpha_{1}\left(s_{1}, s_{2}\right)$ and $\left.\alpha_{2}\left(s_{1}, s_{2}\right)\right)$ by the condition $\nabla v_{N} \cdot \nabla v_{N}=1$, with the inevitable choice of sign of square root being taken in such a way as to guarantee a ray direction propagating away from, and not towards, the boundary $S$.

Having determined $\nabla v_{N}$, the ray directions and equations are now completely determined, along with the solution for $v_{N}$ along them.

\section{$3 \quad$ Friedlander-Keller ray expansions of order $1 / 3$}

Although it is possible to continue with a general development and application of this theory for arbitrary integer $N$, it is perhaps more instructive to use a specific value to illustrate the methodology. The case $N=2$ has already been considered in detail elsewhere [18], and also has the unique property that (19) comprises precisely two terms - one at $O(k)$ and the other at $O\left(k^{1 / 2}\right)$ - and is therefore of identical mathematical structure to the methodology laid out by Friedlander and Keller (take $\alpha=1 / 2$ in their work essentially). For those reasons, and to guarantee a departure from that existing theory, we choose $N=3$.

The field equations to be satisfied by the three phase functions $v_{3}, v_{2}$ and $v_{1}$ and the leading-order amplitude $A_{0}$ follow from (22) and (25), respectively, as

$$
\left|\nabla v_{3}\right|^{2}=1
$$




$$
\begin{aligned}
\nabla v_{2} \cdot \nabla v_{3} & =0 \\
\left|\nabla v_{2}\right|^{2}+2 \nabla v_{1} \cdot \nabla v_{3} & =0 \\
2 i \nabla v_{3} \cdot \nabla A_{0}+A_{0}\left(-2 \nabla v_{1} \cdot \nabla v_{2}+i \nabla^{2} v_{3}\right) & =0 .
\end{aligned}
$$

Two distinct scenarios of natural significance and fundamental importance are when either the geometry of the wavefronts are prescribed or else when appropriate data is prescribed

on a specified curve or surface. In the former case, one example is a given source emitting wavefronts with, say, radial symmetry whilst an example in the latter case might be calculating the field radiating away from a boundary as a result of data specified upon that boundary supplied by a known incident wave.

In both instances, the aim would be to calculate the functional form of the phase and amplitude variations of the radiated fields subject to the above information being provided, and this is the subject of the rest of this section.

\subsection{Prescribed wavefronts}

Prescribing the geometry of a wavefront is mathematically equivalent to prescribing $v_{N}(\boldsymbol{x})$, and we do so for radially symmetric and planar wavefronts, in two and three dimensions in each case, for $N=3$.

\section{Radially-symmetric wavefronts}

Beginning with the two-dimensional case, we set

$$
v_{3}(x, y)=\left(x^{2}+y^{2}\right)^{1 / 2}=r
$$

and our aim is to construct corresponding expressions for $v_{1}, v_{2}$ and $A_{0}$ of the most general form possible. Continuing with plane polar coordinates $(r, \theta),(35)$ now becomes

$$
\frac{\partial v_{2}}{\partial r}=0
$$

so that $v_{2}(r, \theta)=f(\theta)$, where the function $f$ is arbitrary. Equation (36) now yields the partial differential equation

$$
\frac{\left(f^{\prime}(\theta)\right)^{2}}{r^{2}}+2 \frac{\partial v_{1}}{\partial r}=0
$$

for $v_{1}$, which has as its general solution

$$
v_{1}(r, \theta)=\frac{\left(f^{\prime}(\theta)\right)^{2}}{2 r}+g(\theta)
$$

where $g$ is a second arbitrary function. The phase functions $v_{1}$ and $v_{2}$ are now considered to be determined - it is their general functional form that we are interested in - given the initial 
choice (38) for $v_{3}$. In any practical application, the functions $f$ and $g$ would be determined by the prescription of the field on some 'initial wavefront' at $r=a$, say.

Given these solutions so far, we now turn to (37) in order to determine the leading-order amplitude $A_{0}$, and this gives the transport equation

$$
2 i \frac{\partial A_{0}}{\partial r}+A_{0}\left(-2 \frac{\left(f^{\prime}(\theta)\right)^{2} f^{\prime \prime}(\theta)}{r^{3}}-2 \frac{f^{\prime}(\theta) g^{\prime}(\theta)}{r^{2}}+\frac{i}{r}\right)=0,
$$

the solution being found to be

$$
A_{0}(r, \theta)=\frac{D_{1}(\theta)}{r^{1 / 2}} \exp \left[i\left(\frac{\left(f^{\prime}(\theta)\right)^{2} f^{\prime \prime}(\theta)}{2 r^{2}}+\frac{f^{\prime}(\theta) g^{\prime}(\theta)}{r}\right)\right] .
$$

The above expressions for $v_{1}, v_{2}, v_{3}$ and $A_{0}$ are the most general possible for a FriedlanderKeller solution sought subject to the wavefronts $v_{3}=$ constant being specified by (38), and the directivity function $D_{1}(\theta)$ is that that would arise were this to be a standard ray expansion (corresponding to $v_{1} \equiv 0$ and $v_{2} \equiv 0$ ).

As we have suggested earlier in this section, suppose that data is prescribed on $r=a$, say, in the general form

$$
v_{1}(a, \theta)=V_{1}(\theta), \quad v_{2}(a, \theta)=V_{2}(\theta)
$$

for specified functions $V_{1}$ and $V_{2}$. It is then trivial to see that

$$
\begin{aligned}
& f(\theta)=V_{2}(\theta), \\
& g(\theta)=V_{1}(\theta)-\frac{\left(V_{2}^{\prime}(\theta)\right)^{2}}{2 a},
\end{aligned}
$$

and this completes the solution to this boundary value problem.

In three dimensions, we introduce the spherical polar coordinates $(R, \theta, \psi)$ defined by $x=R \sin \theta \cos \psi, y=R \sin \theta \sin \psi, z=R \cos \theta$ and this time set $v_{3}=\left(x^{2}+y^{2}+z^{2}\right)^{1 / 2}=R$ and follow an identical procedure to that followed in the two-dimensional case. This time, (35) reveals that $\frac{\partial v_{2}}{\partial R}=0$, so that

$$
v_{2}(R, \theta, \psi)=F(\theta, \psi)
$$

for some arbitrary function $F$ of the two polar angles alone. Equation (36) can now be re-cast as a partial differential equation for $v_{1}$ which, since $F$ is independent of $R$, can be integrated directly giving

$$
v_{1}(R, \theta, \psi)=\frac{1}{2 R}\left[\left(\frac{\partial F}{\partial \theta}\right)^{2}+\frac{1}{\sin ^{2} \theta}\left(\frac{\partial F}{\partial \psi}\right)^{2}\right]+G(\theta, \psi)
$$

for arbitrary $G$. Using this result, the leading-order transport equation (25) can be written as

$$
\frac{1}{A_{0}} \frac{\partial A_{0}}{\partial R}=i\left[-\frac{\Gamma_{1}(\theta, \psi)}{R^{3}}-\frac{\Gamma_{2}(\theta, \psi)}{R^{2}}+\frac{i}{R}\right],
$$


where

$$
\begin{aligned}
\Gamma_{1}(\theta, \psi) & =\left(\frac{\partial F}{\partial \theta}\right)^{2}\left(\frac{\partial^{2} F}{\partial \theta^{2}}\right)-\frac{\cos \theta}{\sin ^{3} \theta}\left(\frac{\partial F}{\partial \theta}\right)\left(\frac{\partial F}{\partial \psi}\right)^{2} \\
& +\frac{2}{\sin ^{2} \theta}\left(\frac{\partial F}{\partial \theta}\right)\left(\frac{\partial F}{\partial \psi}\right)\left(\frac{\partial^{2} F}{\partial \theta \partial \psi}\right)+\frac{1}{\sin ^{4} \theta}\left(\frac{\partial F}{\partial \psi}\right)^{2}\left(\frac{\partial^{2} F}{\partial \psi^{2}}\right)
\end{aligned}
$$

and

$$
\Gamma_{2}(\theta, \psi)=\left(\frac{\partial F}{\partial \theta}\right)\left(\frac{\partial G}{\partial \theta}\right)+\frac{1}{\sin ^{2} \theta}\left(\frac{\partial F}{\partial \psi}\right)\left(\frac{\partial G}{\partial \psi}\right),
$$

with resulting general solution

$$
A_{0}(R, \theta, \psi)=\frac{D_{2}(\theta, \psi)}{R} \exp \left[i\left(\frac{\Gamma_{1}(\theta, \psi)}{R^{2}}+\frac{\Gamma_{2}(\theta, \psi)}{R}\right)\right] .
$$

The analogue of (44) in this case is to set

$$
v_{1}(a, \theta, \psi)=V_{1}(\theta, \psi), \quad v_{2}(a, \theta, \psi)=V_{2}(\theta, \psi),
$$

where we use the same functional notation since confusion with the results of the previous section is unlikely and where, as in that previous analysis, the functions $V_{1}$ and $V_{2}$ are specified. It is then easy to see that

$$
\begin{aligned}
& F(\theta, \psi)=V_{2}(\theta, \psi) \\
& G(\theta, \psi)=V_{1}(\theta, \psi)-\frac{1}{2 a}\left[\left(\frac{\partial V_{2}}{\partial \theta}\right)^{2}+\frac{1}{\sin ^{2} \theta}\left(\frac{\partial V_{2}}{\partial \psi}\right)^{2}\right]
\end{aligned}
$$

with these expressions, $\Gamma_{1}$ in (50) is obtained simply by setting $F=V_{2}$ whilst $\Gamma_{2}$ in (51) appropriate to this boundary value problem can be expressed in the form

$$
\begin{aligned}
\Gamma_{2}(\theta, \psi) & =\frac{\partial V_{2}}{\partial \theta} \frac{\partial V_{1}}{\partial \theta}+\frac{1}{\sin ^{2} \theta} \frac{\partial V_{2}}{\partial \psi} \frac{\partial V_{1}}{\partial \psi} \\
& -\frac{1}{2 a}\left(\frac{\partial V_{2}}{\partial \theta} \frac{\partial}{\partial \theta}+\frac{1}{\sin ^{2} \theta} \frac{\partial V_{2}}{\partial \psi} \frac{\partial}{\partial \psi}\right)\left(\left(\frac{\partial V_{2}}{\partial \theta}\right)^{2}+\frac{1}{\sin ^{2} \theta}\left(\frac{\partial V_{2}}{\partial \psi}\right)^{2}\right)
\end{aligned}
$$

\section{Planar wavefronts}

Our starting point in two dimensions is to take

$$
v_{3}(x, y)=\lambda x+\mu y
$$

with the constants $\lambda$ and $\mu$ satisfying $\lambda^{2}+\mu^{2}=1$ to ensure that the eikonal equation (34) is satisfied. It then follows almost immediately from (35) that

$$
v_{2}(x, y)=f(\mu x-\lambda y)
$$


for arbitrary $f$, re-using the same functional symbolism as in the two-dimensional radiallysymmetric case since no confusion is likely. The calculations are made simpler by the introduction of new coordinates $\zeta$ and $\xi$ defined by

$$
\begin{aligned}
& \zeta=\mu x-\lambda y \\
& \xi=\lambda x+\mu y,
\end{aligned}
$$

and given these equation (36) then becomes

$$
\left(f^{\prime}(\zeta)\right)^{2}+2 \frac{\partial v_{1}}{\partial \xi}=0
$$

so that

$$
v_{1}(x, y)=-\frac{1}{2} \xi\left(f^{\prime}(\zeta)\right)^{2}+g(\zeta)
$$

for some arbitrary function $g$.

The transport equation (25) is simplified considerably by the fact that $\nabla^{2} v_{3}=0$ in this (and the next) case, and we find that

$$
i \frac{\partial A_{0}}{\partial \xi}+A_{0}\left[\xi\left(f^{\prime}(\zeta)\right)^{2}-f^{\prime}(\zeta) g^{\prime}(\zeta)\right]=0,
$$

leading to the general expression for the leading-order amplitude in terms of $\zeta$ and $\xi$ as

$$
A_{0}(\zeta, \xi)=A_{0}(\zeta, 0) \exp \left[\frac{i}{2} \xi^{2}\left(f^{\prime}(\zeta)\right)^{2} f^{\prime \prime}(\zeta)-i \xi f^{\prime}(\zeta) g^{\prime}(\zeta)\right],
$$

and the solution is complete.

Notice that we have chosen to give data on $\xi=0$; other options are possible and lead to a different representation of the arbitrary 'function of integration' that occurs when solving (63).

The three-dimensional case is slightly more involved and begins by assuming the form

$$
v_{3}(x, y, z)=\lambda x+\mu y+\nu z,
$$

and the constraint $\lambda^{2}+\mu^{2}+\nu^{2}=1$ guarantees that (34) is satisfied. Guided by the previous calculation, we introduce orthogonal coordinates $\xi_{1}, \xi_{2}$ and $\xi_{3}$ - corresponding to a rotation of the original coordinate axes - defined by

$$
\begin{aligned}
& \xi_{1}=\lambda x+\mu y+\nu z \\
& \xi_{2}=-\sqrt{\left(1-\lambda^{2}\right)} x+\frac{\lambda \mu}{\sqrt{\left(1-\lambda^{2}\right)}} y+\frac{\lambda \nu}{\sqrt{\left(1-\lambda^{2}\right)}} z \\
& \xi_{3}=-\frac{\nu}{\sqrt{\left(1-\lambda^{2}\right)}} y+\frac{\mu}{\sqrt{\left(1-\lambda^{2}\right)}} z ;
\end{aligned}
$$

it is easy to check that the matrix representation of this coordinate transformation is orthogonal with determinant +1 . 
With respect to the $\xi_{i}$ 's, (35) becomes simply

$$
\frac{\partial v_{2}}{\partial \xi_{1}}=0
$$

so that $v_{2}\left(\xi_{1}, \xi_{2}, \xi_{3}\right)=F\left(\xi_{2}, \xi_{3}\right)$ for arbitrary function $F$ (and once again we re-use the notation set out in the radially-symmetric case, since confusion is unlikely). Also, after some calculation, (36) can be expressed as

$$
2 \frac{\partial v_{1}}{\partial \xi_{1}}+\left(\frac{\partial F}{\partial \xi_{2}}\right)^{2}+\left(\frac{\partial F}{\partial \xi_{3}}\right)^{2}=0
$$

which can be integrated directly, since $\frac{\partial F}{\partial \xi_{1}}=0$, to give the general expression for $v_{1}$ in the form

$$
v_{1}\left(\xi_{1}, \xi_{2}, \xi_{3}\right)=-\frac{1}{2} \xi_{1}\left[\left(\frac{\partial F}{\partial \xi_{2}}\right)^{2}+\left(\frac{\partial F}{\partial \xi_{3}}\right)^{2}\right]+G\left(\xi_{2}, \xi_{3}\right)
$$

for arbitrary $G$.

This just leaves (25) to solve and, after some algebra, we find that it can be re-written as

$$
\begin{aligned}
\frac{1}{A_{0}} \frac{\partial A_{0}}{\partial \xi_{1}} & =i \xi_{1}\left[\left(\frac{\partial F}{\xi_{2}}\right)^{2}\left(\frac{\partial^{2} F}{\partial \xi_{2}^{2}}\right)+\left(\frac{\partial F}{\xi_{3}}\right)^{2}\left(\frac{\partial^{2} F}{\partial \xi_{3}^{2}}\right)+2\left(\frac{\partial F}{\partial \xi_{2}}\right)\left(\frac{\partial F}{\partial \xi_{3}}\right)\left(\frac{\partial^{2} F}{\partial \xi_{2} \xi_{3}}\right)\right] \\
& -i\left(\frac{\partial F}{\partial \xi_{2}} \frac{\partial G}{\partial \xi_{2}}+\frac{\partial F}{\partial \xi_{3}} \frac{\partial G}{\partial \xi_{3}}\right),
\end{aligned}
$$

and since both $F$ and $G$ are independent of $\xi_{1}$ this is amenable to direct integration, yielding

$$
A_{0}\left(\xi_{1}, \xi_{2}, \xi_{3}\right)=A_{0}\left(0, \xi_{2}, \xi_{3}\right) \exp \left(\frac{i \xi_{1}^{2}}{2} H_{1}\left(\xi_{2}, \xi_{3}\right)-i \xi_{1} H_{2}\left(\xi_{2}, \xi_{3}\right)\right)
$$

where

$$
H_{1}\left(\xi_{2}, \xi_{3}\right)=\left(\frac{\partial F}{\partial \xi_{2}}\right)^{2} \frac{\partial^{2} F}{\partial \xi_{2}^{2}}+\left(\frac{\partial F}{\partial \xi_{3}}\right)^{2} \frac{\partial^{2} F}{\partial \xi_{3}^{2}}+2 \frac{\partial F}{\partial \xi_{2}} \frac{\partial F}{\partial \xi_{3}} \frac{\partial^{2} F}{\partial \xi_{2} \partial \xi_{3}}
$$

and

$$
H_{2}\left(\xi_{2}, \xi_{3}\right)=\frac{\partial F}{\partial \xi_{2}} \frac{\partial G}{\partial \xi_{2}}+\frac{\partial F}{\partial \xi_{3}} \frac{\partial G}{\partial \xi_{3}}
$$

\subsection{Radiation from prescribed boundary data}

Having discussed canonical examples in which the wavefronts are specified we now discuss a different class of problems in which the entire field, its normal derivative, or perhaps some other combination of these quantities, is specified on a given boundary. We shall assume that this specification allows the boundary data for the phase functions and leading-order amplitude of the corresponding radiated field under investigation to be read off directly, 
or else calculated from it. In this account, we restrict attention to the two-dimensional situation, although extension to three dimensions is possible. More specifically, we assume that information contained within the boundary data

$$
v_{3}=v_{3}^{(0)}(s), v_{2}=v_{2}^{(0)}(s), v_{1}=v_{1}^{(0)}(s) \quad \text { on } \quad x=x_{0}(s), y=y_{0}(s)
$$

is given for the phase functions, where the boundary curve $x=x_{0}(s), y=y_{0}(s)$ is parametrised by arc-length $s$, and that appropriate amplitude boundary data is supplied too - we shall return to this point at a later stage of the calculation.

Adopting the two-dimensional equivalent of the procedure following (32) for the determination of $\nabla v_{3}$ and introducing the standard notation $\nabla v_{3}(s)=\left(p_{0}(s), q_{0}(s)\right)$, we find that

$$
\begin{aligned}
& p_{0}(s)=v_{3}^{(0)^{\prime}}(s) x_{0}^{\prime}(s)-\left[1-\left(v_{3}^{(0)^{\prime}}(s)\right)^{2}\right]^{1 / 2} y_{0}^{\prime}(s) \\
& q_{0}(s)=v_{3}^{(0)^{\prime}}(s) y_{0}^{\prime}(s)+\left[1-\left(v_{3}^{(0)^{\prime}}(s)\right)^{2}\right]^{1 / 2} x_{0}^{\prime}(s) .
\end{aligned}
$$

This information gives the direction of the ray leaving the boundary at the point $\left(x_{0}(s), y_{0}(s)\right)$, and we know that

$$
v_{3}(s, \tau)=v_{3}^{(0)}(s)+\tau
$$

along it.

Concerning the other phase functions, we see from (35) and (27) that $\frac{d v_{2}}{d \tau}=0$ along each ray, so that

$$
v_{2}(s, \tau) \equiv v_{2}^{(0)}(s),
$$

whilst (36) then gives the ordinary differential equation

$$
\frac{d v_{1}}{d \tau}=-\frac{1}{2}\left(v_{2}^{(0)^{\prime}}(s)\right)^{2}(\nabla s)^{2}
$$

along each ray, subject to the initial condition $v_{1}(s, 0)=v_{1}^{(0)}(s)$, and where the following relevant expressions are noted:

$$
\begin{gathered}
\nabla s=\frac{\left(q_{0}(s),-p_{0}(s)\right)}{\left(q_{0}(s) p_{0}^{\prime}(s)-p_{0}(s) q_{0}^{\prime}(s)\right)(\tau+\beta(s))}, \\
\beta(s)=\frac{q_{0}(s) x_{0}^{\prime}(s)-p_{0}(s) y_{0}^{\prime}(s)}{q_{0}(s) p_{0}^{\prime}(s)-p_{0}(s) q_{0}^{\prime}(s)} \\
q_{0}(s) x_{0}^{\prime}(s)-p_{0}(s) y_{0}^{\prime}(s)=\left[1-\left(v_{3}^{(0)}(s)\right)^{2}\right]^{1 / 2}
\end{gathered}
$$

and

$$
q_{0}(s) p_{0}^{\prime}(s)-p_{0}(s) q_{0}^{\prime}(s)=\frac{v_{3}^{(0)^{\prime \prime}}(s)}{\left[1-\left(v_{3}^{(0)^{\prime}}(s)\right)^{2}\right]^{1 / 2}}-\kappa_{0}(s)
$$


where $\kappa_{0}(s)$ is the curvature of the boundary. Using these results, it is straightforward to integrate (81) along a ray (taking care to note that there is a $\tau$-dependence on the right-hand-side via $\nabla s$ ) given in (82), and the solution is

$$
\begin{aligned}
v_{1}(s, \tau) & =\frac{\left(v_{2}^{(0)^{\prime}}(s)\right)^{2}}{2\left(q_{0}(s) p_{0}^{\prime}(s)-p_{0}(s) q_{0}^{\prime}(s)\right)^{2}(\tau+\beta(s))}-\frac{\left(v_{2}^{(0)^{\prime}}(s)\right)^{2}}{2\left(q_{0}(s) p_{0}^{\prime}(s)-p_{0}(s) q_{0}^{\prime}(s)\right)^{2} \beta(s)} \\
& +v_{1}^{(0)}(s),
\end{aligned}
$$

and now all three phase functions $v_{1}, v_{2}$ and $v_{3}$ are fully determined, leaving just the leadingorder amplitude $A_{0}(s, \tau)$ to be found. Another very lengthy calculation based around (25) shows that the 'ray' ordinary differential equation satisfied by $A_{0}$ is

$$
2 i \frac{d A_{0}}{d \tau}+A_{0}\left[\frac{i}{\tau+\beta(s)}-\frac{2 v_{2}^{(0)^{\prime}}(s)\left(\frac{\partial v_{1}}{\partial s}-v_{3}^{(0)^{\prime}}(s) \frac{\partial v_{1}}{\partial \tau}\right)}{\left(p_{0}^{\prime}(s) q_{0}(s)-p_{0}(s) q_{0}^{\prime}(s)\right)^{2}(\tau+\beta(s))^{2}}\right]=0
$$

with $v_{1}$ given by (86), and further involved calculation shows that this can be re-expressed in the more concise form

$$
\frac{d A_{0}}{d \tau}+A_{0} \sum_{j=1}^{4} \frac{\sigma_{j}(s)}{(\tau+\beta(s))^{j}}=0
$$

where

$$
\begin{aligned}
\sigma_{1}(s) & =\frac{1}{2} \\
\sigma_{2}(s) & =\frac{i v_{2}^{(0)^{\prime}}(s)}{\left(p_{0}^{\prime}(s) q_{0}(s)-p_{0}(s) q_{0}^{\prime}(s)\right)^{2}} \\
& \times\left[v_{1}^{(0)^{\prime}}(s)-\frac{1}{2} \frac{d}{d s}\left(\frac{1}{\beta(s)}\left(\frac{v_{2}^{(0)^{\prime}}(s)}{p_{0}^{\prime}(s) q_{0}(s)-p_{0}(s) q_{0}^{\prime}(s)}\right)^{2}\right)\right] \\
\sigma_{3}(s) & =\frac{i v_{2}^{(0)^{\prime}}(s)}{2\left(p_{0}^{\prime}(s) q_{0}(s)-p_{0}(s) q_{0}^{\prime}(s)\right)^{2}} \frac{d}{d s}\left(\left(\frac{v_{2}^{\prime}(s)}{p_{0}^{\prime}(s) q_{0}(s)-p_{0}(s) q_{0}^{\prime}(s)}\right)^{2}\right) \\
\sigma_{4}(s) & =\frac{i\left(v_{2}^{(0)^{\prime}}(s)\right)^{3}\left(v_{3}^{(0)^{\prime}}(s)-\beta^{\prime}(s)\right)}{2\left(p_{0}^{\prime}(s) q_{0}(s)-p_{0}(s) q_{0}^{\prime}(s)\right)^{4}} .
\end{aligned}
$$

This differential equation is easily solved and the final expression is

$$
A_{0}(s, \tau)=A_{0}(s, 0)\left(\frac{\beta(s)}{\tau+\beta(s)}\right)^{1 / 2} \exp \left[\sum_{j=2}^{4} \frac{\sigma_{j}(s)}{(j-1)}\left(\frac{1}{(\tau+\beta(s))^{j-1}}-\frac{1}{(\beta(s))^{j-1}}\right)\right]
$$

results, completing the explicit construction of the leading-order Friedlander-Keller ray expansion in this case. 


\section{Scattering from a class of perturbed, curved boundaries}

We conclude the main body of the paper by applying the results just derived to a generalisation of the class of scattering problems referred to in the Introduction and Motivation section of this paper, with this application in its own right being a major motivation for this current study of Friedlander-Keller ray expansions. In that original class of problems, the incoming wave had an asymptotic representation given to leading-order by (15), which (to this order) could have been written in the form

$$
\phi^{i n c}(x, y) \sim F(x, y) e^{i k u(x, y)},
$$

where $F(x, y)$ is obtained from $F_{0}(\Theta)$ in $(15)$ using the relation $\tan \Theta=(y+h) / x$. More specifically, we take

$$
u(x, y)=R(x, y)=\left[x^{2}+(y-h)^{2}\right]^{1 / 2}
$$

and

$$
F(x, y)=\left[x^{2}+(y-h)^{2}\right]^{-1 / 4} F_{0}\left(\tan ^{-1}\left(\frac{y+h}{x}\right)\right) .
$$

Our first generalisation is to leave $F(x, y)$ and $u(x, y)$ as being generic (but specified in any given application) with $u$ satisfying the eikonal equation (6) and $F$ the associated leading-order transport equation (9), with $F$ replacing $A_{0}$. Taking $F$ and $u$ as given by the expressions immediately above then permits the reflection of a general cylindrical wave to be analysed, whilst setting

$$
u(x, y)=x \cos \theta-y \sin \theta
$$

and

$$
F(x, y)=F_{1}(x+y \cot \theta)
$$

would cover the case of plane wave incidence with arbitrary amplitude profile dictated by the function $F_{1}$.

Our second generalisation concerns the nature of the perturbed scattering boundary itself; the Introduction contained reference to the boundary $y=k^{-1 / N} f(x)$, to be considered as a small deviation away from the flat boundary $y=0$. Here, although we do specify $N=3$ (keeping $N$ general is possible, but the salient features of the calculations are best exposed by using a particular value of $N$ ) we now consider small deviations away from an arbitrarily curved boundary $x=x_{0}(s), y=y_{0}(s)$ (with $s$ being arc-length along it) in the given form

$$
x=x_{0}(s)+k^{-1 / 3} \hat{x}_{0}(s), y-y_{0}(s)+k^{-1 / 3} \hat{y}_{0}(s)
$$

in which $x_{0}, y_{0}, \hat{x}_{0}$ and $\hat{y}_{0}$ are all given functions (although, of course, $s$ is no longer arclength along this perturbed curve). We also note that setting

$$
x_{0}(s)=s, y_{0}(s)=0, \hat{x}_{0}(s)=0, \hat{y}_{0}(s)=f(s)
$$


reproduces the original specification $y=k^{-1 / 3} f(x)$ for this particular choice of $N$.

Adopting the leading-order ansatz

$$
\phi^{r e f} \sim A_{0} e^{i k v_{3}+i k^{2 / 3} v_{2}+i k^{1 / 3} v_{1}}
$$

in Friedlander-Keller format for the reflected field, we find that adopting a Neumann boundary condition to be applied to the total field $\phi^{\text {tot }}=\phi^{i n c}+\phi^{r e f}$ at the perturbed boundary yields, exactly,

$$
\begin{array}{r}
{\left[k\left(x_{0}^{\prime}+k^{-1 / 3} \hat{x}_{0}^{\prime}\right) \frac{\partial u}{\partial y}-k\left(y_{0}^{\prime}+k^{-1 / 3} \hat{y}_{0}^{\prime}\right) \frac{\partial u}{\partial x}\right] F e^{i k u}+} \\
{\left[\left(x_{0}^{\prime}+k^{-1 / 3} \hat{x}_{0}^{\prime}\right)\left(\sum_{m=1}^{3} k^{m / 3} \frac{\partial v_{m}}{\partial y}\right)-\left(y_{0}^{\prime}+k^{-1 / 3} \hat{y}_{0}^{\prime}\right)\left(\sum_{m=1}^{3} k^{m / 3} \frac{\partial v_{m}}{\partial x}\right)\right] A_{0} e^{i \sum_{m=1}^{3} k^{m / 3} v_{m}+}+} \\
{\left[\left(x_{0}^{\prime}+k^{-1 / 3} \hat{x}_{0}^{\prime}\right) \frac{\partial F}{\partial y}-\left(y_{0}^{\prime}+k^{-1 / 3} \hat{y}_{0}^{\prime}\right) \frac{\partial F}{\partial x}\right] e^{i k u}+} \\
{\left[\left(x_{0}^{\prime}+k^{-1 / 3} \hat{x}_{0}^{\prime}\right) \frac{\partial A_{0}}{\partial y}-\left(y_{0}^{\prime}+k^{-1 / 3} \hat{y}_{0}^{\prime}\right) \frac{\partial A_{0}}{\partial x}\right] e^{i \sum_{m=1}^{3} k^{m / 3} v_{m}}=0 .}
\end{array}
$$

All functions arising in the above boundary condition are to be evaluated on the perturbed boundary $x=x_{0}(s)+k^{-1 / 3} \hat{x}_{0}(s), y=y_{0}(s)+k^{-1 / 3} \hat{y}_{0}(s)$ and then re-expanded via the use of a Taylor series, a procedure which amounts to a linearisation of the boundary condition onto the unperturbed boundary $x=x_{0}(s), y=y_{0}(s)$. Thus, the leading-order terms in the coefficients of $(102)$ arise at $O(k)$ with the next terms being $O\left(k^{2 / 3}\right)$, these coming not just from the terms $\sum_{m=1}^{3} k^{m / 3} \frac{\partial v_{m}}{\partial x}$ and $\sum_{m=1}^{3} k^{m / 3} \frac{\partial v_{m}}{\partial y}$ but also from the leading-order terms in the Taylor series expansions of terms like $i k x_{0}^{\prime} F \frac{\partial u}{\partial y}$, for example. These are essentially amplitude considerations, and whilst they are vital when considering higher-order terms in the amplitude of the reflected field they do not concern us at all here, since we restrict attention to the determination of the leading-order terms only.

However, this general feature $i s$ something that must be accounted for in the evaluation of the boundary data for the phase functions $v_{1}, v_{2}$ and $v_{3}$. For example, the $O\left(k^{-1 / 3}\right)$ and $O\left(k^{-2 / 3}\right)$ corrections to $u$ will, when multiplied by $k$, lead to terms of $O\left(k^{2 / 3}\right)$ and $O\left(k^{1 / 3}\right)$, respectively, in the boundary evaluation of the overall phase. As such, these terms will therefore contribute to the boundary data for $v_{2}$ and $v_{1}$, respectively; similarly, such corrections to $v_{2}$ will affect the data for $v_{1}$ whilst all three phase terms have $O(1)$ contributions which become part of the overall $O(1)$ contribution involving $F$ and $A_{0}$ to be obtained from (102) as part of the amplitude considerations mentioned previously.

Hence, the order in which information is extracted from (102) is crucial: 'phase' data in the exponents is to be determined first of all, leading initially to data for $v_{3}$ and which can be used to solve for $v_{3}$ once and for all. Given this, this general procedure is followed for $v_{2}$ 
and then for $v_{1}$. Now that all three phase functions are completely determined, their contributions at $O(1)$ (along with equivalent terms generated by the incident field) are retained in (102) in order to determine the boundary data for the leading-order amplitude $A_{0}$ of the reflected field.

We begin this procedure by writing

$$
i k u(x, y)=i \sum_{m=0}^{3} k^{m / 3} u_{m}(s)+O\left(k^{-1 / 3}\right)
$$

as the expansion for $u$ when evaluated on (99), emphasising that each function $u_{m}(s)$ $(m=0, \ldots, 3)$ is known (since $u$ is). Next, we equate terms involving like powers of $k^{m / 3}$ $(m=1,2,3)$ in both of the separate exponentials in (102), yielding the general expression

$$
u_{m}(s)=\sum_{p \geq m}^{3} \frac{1}{(p-m) !}\left(\hat{x}_{0} \frac{\partial}{\partial x}+\hat{y}_{0} \frac{\partial}{\partial y}\right)^{p-m} v_{p}(s, 0), \quad m=3,2,1,
$$

where we deliberately consider this equation in descending numerical order for $m$ for the reasons mentioned previously, and where the differential operator $\left(\hat{x}_{0} \frac{\partial}{\partial x}+\hat{y}_{0} \frac{\partial}{\partial y}\right)^{p-m} v_{p}$ is just the function $v_{m}$ itself when $p=m$. To make all of this procedure more transparent, and switching to the ray coordinates $s$ and $\tau$ in which these calculations are perhaps best performed, we re-express (104) in the form

$$
\begin{aligned}
v_{m}^{(0)}(s) & =u_{m}(s) \\
& -\sum_{p: p>m}^{3} \frac{1}{p !}\left[\frac{\left(\hat{x}_{0} q_{0}-\hat{y}_{0} p_{0}\right) \frac{\partial}{\partial s}-\left[\left(\hat{x}_{0} y_{0}^{\prime}-\hat{y}_{0} x_{0}^{\prime}\right)+\tau\left(\hat{x}_{0} q_{0}^{\prime}-\hat{y}_{0} p_{0}^{\prime}\right)\right] \frac{\partial}{\partial \tau}}{\left(q_{0} x_{0}^{\prime}-p_{0} y_{0}^{\prime}\right)+\tau\left(p_{0}^{\prime} q_{0}-p_{0} q_{0}^{\prime}\right)}\right]^{p-m} v_{p}(1)
\end{aligned}
$$

valid for $m=3,2,1$ and where the sum is to be evaluated on the boundary $\tau=0$ once the differential operator has been applied. This formulation also shows how the solution for $v_{3}$ feeds into the calculation of that for $v_{2}$, and so on. In fact, once this procedure has been followed for $m=3,2,1$ we have then calculated the data for all three phase functions, which can then be fed into their general solutions and the phase aspect of the determination of the reflected field is considered complete, leaving just the amplitude to be finalised.

A formula for $A_{0}(s, \tau)$ is presented in (93), together with equations (89)-(92) immediately preceeding it, and (83). The expressions for $p_{0}$ and $q_{0}$ in those various formulae are given in (77) and (78), respectively, which in turn rely upon the data for $v_{3}$ just calculated. What remains to be found is $A_{0}(s, 0)$ and in view of the previous discussion, this follows from (102) as

$$
A_{0}(s, 0)=F\left(x_{0}(s), y_{0}(s)\right) \exp \left[i u_{0}(s)-i \sum_{p=1}^{3} \frac{1}{p !}\left(\hat{x}_{0}(s) \frac{\partial}{\partial x}+\hat{y}_{0}(s) \frac{\partial}{\partial y}\right)^{p} v_{p}(s, 0)\right],
$$


a result which requires the fact that the ratio of the value of $q_{0}(s) x_{0}^{\prime}(s)-p_{0}(s) y_{0}^{\prime}(s)$ relevant to the incident field to that for the reflected field is precisely -1 . This is for Neumann data, and the equivalent result for Dirichlet data is simply the negative of that in (106).

This expression for the leading-order amplitude is in closed, analytical form with all quantities within it either given directly, or easily calculable from, the theory presented earlier in this section. However, it turns out that it can be simplified further still by using the field equations (34)-(36) to eliminate, for example, the $y$-derivatives occurring in the summation within the exponent on the right-hand-side of (106). For example, appropriate differentiation of (34) yields

$$
\frac{\partial^{2} v_{3}}{\partial x^{2} \partial y}=-\frac{1}{q_{0}^{3}}\left(\frac{\partial^{2} v_{3}}{\partial x^{2}}\right)^{2}-\frac{p_{0}}{q_{0}} \frac{\partial^{3} v_{3}}{\partial x^{3}}
$$

and

$$
\frac{\partial^{2} v_{3}}{\partial x \partial y^{2}}=\frac{2 p_{0}}{q_{0}^{4}}\left(\frac{\partial^{2} v_{3}}{\partial x^{2}}\right)^{2}+\frac{p_{0}^{2}}{q_{0}} \frac{\partial^{3} v_{3}}{\partial x^{3}},
$$

whilst (35) yields

$$
\frac{\partial v_{2}}{\partial y}=-\frac{p_{0}}{q_{0}} \frac{\partial v_{2}}{\partial x}
$$

with other results of a similar nature following easily. This allows us to re-express the summation in (106) in the form

$$
\begin{aligned}
\sum_{p=1}^{3} \frac{1}{p !}\left(\hat{x}_{0} \frac{\partial}{\partial x}+\hat{y}_{0} \frac{\partial}{\partial y}\right)^{p} & =\sum_{n=1}^{3}\left(\frac{1}{n ! q_{0}^{n}}\right)\left(\frac{\partial^{2} v_{n}}{\partial x^{n}}\right)\left(q_{0} \hat{x}_{0}-p_{0} \hat{y}_{0}\right)^{n} \\
& -\frac{\hat{y}_{0}}{2 q_{0}^{5}}\left[\left(q_{0} \hat{x}_{0}-p_{0} \hat{y}_{0}\right) \frac{\partial^{2} v_{3}}{\partial x^{2}}+q_{0} \frac{\partial v_{2}}{\partial x}\right]^{2}
\end{aligned}
$$

with all quantities to be evaluated (after differentiation) on $\tau=0$.

The apparent asymmetry between $\hat{x}_{0}$ and $\hat{y}_{0}$ introduced by the multiplicative factor of $\hat{y}_{0}$ alone in the final term of (110) is because we have chosen to re-express the coefficients in terms of $x$-derivatives of $v_{1}, v_{2}$ and $v_{3}$; had we chosen to do so in terms of $y$-derivatives then it would be a factor of $\hat{x}_{0}$ alone that appears, albeit adjacent to a different factor. The advantage of this representation is that all we now need do to compute the sum explicitly is evaluate the five derivatives $\frac{\partial^{3} v_{3}}{\partial x^{3}}, \frac{\partial^{2} v_{3}}{\partial x^{2}}, \frac{\partial^{2} v_{2}}{\partial x^{2}}, \frac{\partial v_{2}}{\partial x}$ and $\frac{\partial v_{1}}{\partial x}$, and even then we just need these derivatives evaluated on $\tau=0$. For completeness, and to close the calculation of the leading-order amplitude of the reflected field, these derivatives (all evaluated on $\tau=0$ ) are listed as follows:

$$
\begin{aligned}
\frac{\partial^{3} v_{3}}{\partial x^{3}} & =\frac{1}{\left(q_{0}(s) x_{0}^{\prime}(s)-p_{0}(s) y_{0}^{\prime}(s)\right)}\left[\frac{2 q_{0}^{2}(s) q_{0}^{\prime}(s)}{\beta(s)}-\frac{\beta^{\prime}(s) q_{0}^{3}(s)}{\beta^{2}(s)}+\frac{q_{0}^{2}(s) y_{0}^{\prime}(s)}{\beta^{2}(s)}\right] \\
\frac{\partial^{2} v_{3}}{\partial x^{2}} & =\frac{q_{0}^{2}(s)}{\beta(s)}
\end{aligned}
$$




$$
\begin{aligned}
\frac{\partial^{2} v_{2}}{\partial x^{2}} & =\frac{q_{0}(s)}{\left(q_{0}(s) x_{0}^{\prime}(s)-p_{0}(s) y_{0}^{\prime}(s)\right)} \frac{d}{d s}\left[\frac{q_{0}(s) v_{2}^{(0)^{\prime}}(s)}{\left(q_{0}(s) x_{0}^{\prime}(s)-p_{0}(s) y_{0}^{\prime}(s)\right)}\right] \\
& +\frac{y_{0}^{\prime}(s) q_{0}(s) v_{2}^{(0)^{\prime}}(s)}{\left(q_{0}(s) x_{0}^{\prime}(s)-p_{0}(s) y_{0}^{\prime}(s)\right)^{2}} \\
\frac{\partial v_{2}}{\partial x} & =\frac{q_{0}(s) v_{2}^{(0)^{\prime}}(s)}{\left(q_{0}(s) x_{0}^{\prime}(s)-p_{0}(s) y_{0}^{\prime}(s)\right)} \\
\frac{\partial v_{1}}{\partial x} & =\frac{1}{\left(q_{0}(s) x_{0}^{\prime}(s)-p_{0}(s) y_{0}^{\prime}(s)\right)}\left[q_{0}(s) v_{1}^{(0)^{\prime}}(s)+\frac{y_{0}^{\prime}(s)\left(v_{2}^{(0)^{\prime}}(s)\right)^{2}}{2\left(q_{0}(s) x_{0}^{\prime}(s)-p_{0}(s) y_{0}^{\prime}(s)\right)^{2}}\right]
\end{aligned}
$$

This procedure is completely general and will, in principle at least, apply to any incoming wave incident upon any perturbed boundary within the general class being considered. Of particular interest are those cases associated with plane and cylindrical wave incidence, for which we have already noted that $u(x, y)=x \cos \theta-y \sin \theta$ and $u(x, y)=\left[x^{2}+(y-h)^{2}\right]^{1 / 2}$, respectively; here, the source of the incident cylindrical wave is located at the point with Cartesian coordinates $(0, h)$ with $k h \gg 1$ and we assume that the wavefronts have established their cylindrical symmetry before they reach the reflecting boundary.

We have seen that everything needed to construct the leading-order solution for the reflected field can be obtained once we know the coefficient functions $u_{m}(s)$ in (103) and the boundary data $v_{3}^{(0)}(s), v_{2}^{(0)}(s)$ and $v_{1}^{(0)}(s)$ for the three phase functions $v_{3}, v_{2}$ and $v_{1}$, respectively (where the latter boundary data can being found explicitly from (105)). From these various expressions, the components of the reflected ray directions $\left(p_{0}(s)\right.$ and $\left.q_{0}(s)\right)$ can be determined using the procedure described earlier, whilst (106) can be used to write down the boundary data for the amplitude coefficient. So the recipe is complete and we end this section, and the main part of the paper, by simply listing the necessary ingredients required for each of the plane and cylindrical wave incidence cases as follows, together with the comment that the algebraic manipulation required to obtain some of these results is very involved indeed:

\section{Plane wave incidence}

$$
\begin{aligned}
u_{3}(s) & =x_{0}(s) \cos \theta-y_{0}(s) \sin \theta \\
u_{2}(s) & =\hat{x}_{0}(s) \cos \theta-\hat{y}_{0}(s) \sin \theta \\
u_{1}(s) & =0 \\
u_{0}(s) & =0 \\
v_{3}^{(0)}(s) & =x_{0}(s) \cos \theta-y_{0}(s) \sin \theta \\
v_{2}^{(0)}(s) & =2\left[\hat{x}_{0}(s) y_{0}^{\prime}(s)-\hat{y}_{0}(s) x_{0}^{\prime}(s)\right]\left[x_{0}^{\prime}(s) \sin \theta+y_{0}^{\prime}(s) \cos \theta\right] \\
v_{1}^{(0)}(s) & =-2\left[\hat{x}_{0}^{\prime}(s) y_{0}^{\prime}(s)-\hat{y}_{0}^{\prime}(s) x_{0}^{\prime}(s)\right]\left[q_{0}(s) \hat{x}_{0}(s)-p_{0}(s) \hat{y}_{0}(s)\right]
\end{aligned}
$$




$$
\begin{aligned}
& -\frac{\kappa_{0}(s)\left[q_{0}(s) \hat{x}_{0}(s)-p_{0}(s) \hat{y}_{0}(s)\right]^{2}}{\left(x_{0}^{\prime}(s) \sin \theta+y_{0}^{\prime}(s) \cos \theta\right)} \\
p_{0}(s) & =\left[\left(x_{0}^{\prime}(s)\right)^{2}-\left(y_{0}^{\prime}(s)\right)^{2}\right] \cos \theta-2 x_{0}^{\prime}(s) y_{0}^{\prime}(s) \sin \theta \\
q_{0}(s) & =\left[\left(x_{0}^{\prime}(s)\right)^{2}-\left(y_{0}^{\prime}(s)\right)^{2}\right] \sin \theta+2 x_{0}^{\prime}(s) y_{0}^{\prime}(s) \cos \theta
\end{aligned}
$$

\section{Cylindrical wave incidence}

$$
\begin{aligned}
& u_{3}(s)=\left[x_{0}^{2}(s)+\left(y_{0}(s)-h\right)^{2}\right]^{1 / 2} \\
& u_{2}(s)=\frac{\left[x_{0}(s) \hat{x}_{0}(s)+\left(y_{0}(s)-h\right) \hat{y}_{0}(s)\right]}{\left[x_{0}^{2}(s)+\left(y_{0}(s)-h\right)^{2}\right]^{1 / 2}} \\
& u_{1}(s)=\frac{\left[\left(y_{0}(s)-h\right) \hat{x}_{0}(s)-x_{0}(s) \hat{y}_{0}(s)\right]^{2}}{2\left[x_{0}^{2}(s)+\left(y_{0}(s)-h\right)^{2}\right]^{3 / 2}} \\
& u_{0}(s)=-\frac{\left[x_{0}(s) \hat{x}_{0}(s)+\left(y_{0}(s)-h\right) \hat{y}_{0}(s)\right]\left[\left(y_{0}(s)-h\right) \hat{x}_{0}(s)-x_{0}(s) \hat{y}_{0}(s)\right]^{2}}{2\left[x_{0}^{2}(s)+\left(y_{0}(s)-h\right)^{2}\right]^{5 / 2}}(128 \\
& v_{3}^{(0)}(s)=\left[x_{0}^{2}(s)+\left(y_{0}(s)-h\right)^{2}\right]^{1 / 2} \\
& v_{2}^{(0)}(s)=\frac{2\left[y_{0}^{\prime}(s) \hat{x}_{0}(s)-x_{0}^{\prime}(s) \hat{y}_{0}(s)\right]\left[x_{0}(s) y_{0}^{\prime}(s)-\left(y_{0}(s)-h\right) x_{0}^{\prime}(s)\right]}{\left[x_{0}^{2}(s)+\left(y_{0}(s)-h\right)^{2}\right]^{1 / 2}} \\
& v_{1}^{(0)}(s)=\frac{2\left[x_{0}(s) x_{0}^{\prime}(s)+\left(y_{0}(s)-h\right) y_{0}^{\prime}(s)\right]^{2}\left[\hat{x}_{0}(s) y_{0}^{\prime}(s)-\hat{y}_{0}(s) x_{0}^{\prime}(s)\right]^{2}}{\left[x_{0}^{2}(s)+\left(y_{0}(s)-h\right)^{2}\right]^{3 / 2}} \\
& -\frac{\kappa_{0}(s)\left[x_{0}^{2}(s)+\left(y_{0}(s)-h\right)^{2}\right]^{1 / 2}\left(q_{0}(s) \hat{x}_{0}(s)-p_{0}(s) \hat{y}_{0}(s)\right)^{2}}{\left(x_{0}(s) y_{0}^{\prime}(s)-\left(y_{0}(s)-h\right) x_{0}^{\prime}(s)\right)} \\
& \text { - } 2\left(y_{0}^{\prime}(s) \hat{x}_{0}^{\prime}(s)-x_{0}^{\prime}(s) \hat{y}_{0}^{\prime}(s)\right)\left(q_{0}(s) \hat{x}_{0}(s)-p_{0}(s) \hat{y}_{0}(s)\right) \\
& p_{0}(s)=\frac{\left[\left(\left(x_{0}^{\prime}(s)\right)^{2}-\left(y_{0}^{\prime}(s)\right)^{2}\right) x_{0}(s)+2 x_{0}^{\prime}(s) y_{0}^{\prime}(s)\left(y_{0}(s)-h\right)\right]}{\left[x_{0}^{2}(s)+\left(y_{0}(s)-h\right)^{2}\right]^{1 / 2}} \\
& q_{0}(s)=\frac{\left[2 x_{0}^{\prime}(s) y_{0}^{\prime}(s) x_{0}(s)-\left(\left(x_{0}^{\prime}(s)\right)^{2}-\left(y_{0}^{\prime}(s)\right)^{2}\right)\left(y_{0}(s)-h\right)\right]}{\left[x_{0}^{2}(s)+\left(y_{0}(s)-h\right)^{2}\right]^{1 / 2}} .
\end{aligned}
$$

\section{$5 \quad$ Discussion and concluding remarks}

In this paper we have concentrated on Friedlander-Keller ray expansions in which the exponent of the ray ansatz contains terms proportional to all powers of $k^{1 / N}$ (for positive integer $N$ ) between and including $k^{1 / N}$ and $k$; the original work of Friedlander and Keller 
would have contained just one such term (additional to that in $k$ ) proportional to $k^{\alpha}$ with $\alpha=m / N$ for one particular integer $m$ in the range $1 \leq m \leq N-1$, though that analysis was actually valid for any $\alpha$, rational or otherwise.

We have concentrated in particular detail on the case $N=3$, though the methods that we have used to solve that case are equally applicable to other larger choices of $N$. There do not appear to be generic (in $N$ ) solutions for the phase functions $v_{1}, v_{2}, \ldots, v_{N}$ and it seems that each choice of $N$ must be treated separately, with the case $N=3$ considered here acting as an excellent template and paradigm.

All of this has been done for scalar field variables and, as such, is relevant to vectorial circumstances (such as applications in elasticity and electromagnetism) only when those fields can be described in terms of their components or else a scalar potential function. This is not always the case, and so a natural and important extension of the theoretical developments considered here is to modify the underlying Friedlander-Keller ansatz into vectorial form itself and then to apply it directly to Navier's equations of linear elasticity or Maxwell's equations of electromagnetism. Pushing aside (though one should not) the inherent difficulties in these being vector-valued field equations, added complications arise from the presence of two different types of wave motion in each case ('longitudinal' and 'shear' in elasticity, 'electric' and 'magnetic' in electromagnetism); in the former case this is complicated further by the fact that these waves have different wave speeds, and hence different wavenumbers, and so each constituent wave type requires its own Friedlander-Keller ray expansion. The latter (electromagnetic) case does not have this feature (all wave speeds are that of light, of course) but instead has the additional equations $\boldsymbol{\nabla} \cdot \boldsymbol{E}=\mathbf{0}, \boldsymbol{\nabla} \cdot \boldsymbol{B}=\mathbf{0}$ (in standard notation) to be satisfied in source-free, charge-free space. These are, of course, part of Maxwell's equations and can be used to obtain wave equations for $\boldsymbol{E}$ and $\boldsymbol{B}$ alone, but whichever approach is taken $\boldsymbol{E}$ and $\boldsymbol{B}$ are ultimately coupled and this must become a feature of a Friedlander-Keller ray analysis not present in the calculations performed here. The preceding comments pertain to the derivation of the generic field equations. In problems involving reflection of waves at boundaries, for example, then we would also have to accommodate mode conversion phenomena where, in either an elastic or electromagnetic context, a wave of one type incident upon a free boundary generates reflected waves of both types. Once more, this feature is missing from the current theory and so further illustrates the need for a vectorial theory which does encapsulate it.

Returning to the scalar case, there are various important extensions to both the theoretical development of the method and its applications. For instance, problems in which the wave speed of propagation is not constant, so that the rays associated with the incident and reflected fields alike are no longer straight lines, is one possibility whilst fully timedependent (rather than simply time-harmonic, as considered here) problems is another. In this second case, the key equation for the principal phase term analogous to $v_{3}$ is no longer the eikonal equation but is instead the time-dependent version

$$
\left(\nabla v_{N}\right)^{2}=\left(\frac{\partial v_{N}}{\partial t}\right)^{2}
$$


and fundamental aspects of the ray geometry, and the solution to the transport equations for the time-dependent amplitudes along the rays, are now significantly different; of course, the field equations, and hence also the corresponding solutions, for the other phase functions must be modified similarly.

Concerning the application to the scattering of monochromatic waves at perturbed boundaries discussed in the second half of the paper, we remark that other two-dimensional perturbation profiles that we could examine include the more general class

$$
\boldsymbol{x}=\boldsymbol{x}_{\mathbf{0}}(s)+k^{-\lambda / N} \hat{\boldsymbol{x}}_{\mathbf{0}}(s), \quad \lambda=1,2, \ldots N-1
$$

in this paper we have restricted attention to the case $\lambda=1, N=3$ but other more general values are just as important and they can all be treated using the methodology presented within this paper, as can an extension of the entire theory to encompass two-dimensional boundaries in a three-dimensional setting.

The final example that we cite as worthy of further study concerns reflection and transmission at curved, perturbed boundaries between two distinct wave-bearing media. As we have mentioned previously in the context of elastic waves, two wavenumbers are now involved and whilst the individual Friedlander-Keller ray expansions for each of the two media are to be treated exactly as presented in this paper, complications arise when 'phase matching' their exponents when satisfying the boundary conditions at the common interface. The fact that the wavenumbers are different turns out to be crucial and introduces significant difficulties when calculating the boundary data for the phase functions of both expansions (one for each medium) when linearised onto the underlying unperturbed boundary. There is also the possibility that one of the transmitted rays in the second medium is launched in a direction locally tangent to the unperturbed boundary, and so does not radiate information by itself into the bulk of that medium. That is to say, total internal reflection has occurred which could, in turn, lead to the excitation of a whispering gallery mode, together with subsequent re-radiation back into the first medium supporting the incident wave via a so-called 'head' or 'lateral' wave.

Such an occurrence is a diffractive phenomenon, and we end by commenting that all possible diffraction effects such as arise when an incident ray (a) is tangent to the reflecting boundary (b) excites a radiative 'free-mode' of the interfacial boundary condition or (c) impinges upon a point of non-analyticity of either the boundary itself or of the data prescribed upon it, for example, are avoided in this current account.

These aspects, together with those reviewed previously, are all under investigation and will be reported upon separately.

\section{References}

[1] FRIEDLANDER, F. G. \& KELLER, J. B. (1955) Asymptotic solutions of $\left(\nabla^{2}+k^{2}\right) u=0$. Commun. Pure Appl. Math. 6, 387-394. 
[2] LUNEBERG, R. K. (1965) Mathematical Theory of Optics. Cambridge University Press, UK.

[3] KLINE, M. \& KAY, I. W. (1965) Electromagnetic Theory and Geometrical Optics. John Wiley and Sons, Interscience: New York.

[4] KELLER, J. B. (1962) Geometrical theory of diffraction. J. Opt. Soc. Am. A 52, $116-130$.

[5] BLEISTEIN, N. (1984) Mathematical Methods for Wave Phenomena. Academic Press, Orlando.

[6] KELLER, J. B. \& LEWIS, R. M. (1995) Asymptotic methods for partial differential equations: the reduced wave equation and Maxwell's equations. In: J. B. Keller, D. W. McLaughlin \& G. C. Papanicolaou (editors), Surveys in Applied Mathematics, Vol. 1, Plenum Press, New York, 1-82.

[7] KELLER, J. B., LEWIS, R. M. \& SECKLER, B. D. (1956) Asymptotic solutions of some diffraction problems. Commun. Pure Appl. Math. 9, 207-265.

[8] Molinet, F., ANDronov, I. \& BOUCHE, D. (2005) Asymptotic and Hybrid Methods in Electromagnetics. IEE Electromagnetic Waves Series, 51, The Institution of Electrical Engineers.

[9] ACHEnBACH, J. D., GAUTESEN, A. K. \& McMAKEN, H. (1982) Ray Methods for Waves in Elastic Solids. Pitman Publishing, Inc., Massachusetts.

[10] LEONTOVICH, M. \& FOCK, V. (1945) Solution of the problem of propagation of electromagnetic waves along the Earth's surface by the method of the parabolic equation. J. Phys. 10, 13-24.

[11] LEVY, B. R. \& KELLER, J. B. (1959) Diffraction by a smooth object. Commun. Pure Appl. Math. 12, 159-209.

[12] TEW, R. H., CHAPMAN, S. J., KING, J. R., OCKENDON, J. R., SMITH, B. J. \& ZAFARULLAH, I. (2000) Scalar wave diffraction by tangent rays. Wave Motion 32, 363-380.

[13] LEWIS, R. M., BLEISTEIN, N. \& LUDWIG, D. (1967) Uniform asymptotic theory of creeping waves. Commun. Pure Appl. Math. 20, 295-328.

[14] RULF, B. (1968) Uniform asymptotic theory of diffraction at an interface. Commun. Pure Appl. Math. 21, 67-76.

[15] LUDWIG, D. (1966) Uniform asymptotic expansions at a caustic. Commun. Pure Appl. Math. 19, 215-250. 
[16] BUCHAL, R. N. \& KELLER, J. B. (1960) Boundary layer problems in diffraction theory. Commun. Pure Appl. Math. 13, 85-114.

[17] ABBOUD, T. \& AMMARI, H. (1996) Diffraction at a curved grating: approximation by an infinite plane grating J. Math. Anal. Appl. 202, 1076-1100.

[18] TEW, R. H. (2018) Friedlander-Keller ray expansions and scalar wave reflection at canonically-perturbed boundaries. Euro. J. Appl. Math. doi:10.1017/S0956792517000353, 1-22. 